\title{
Functional Monadic Bounded Algebras
}

\author{
Robert Goldblatt
}

9 June 2010

\begin{abstract}
The variety MBA of monadic bounded algebras consists of Boolean algebras with a distinguished element $E$, thought of as an existence predicate, and an operator $\exists$ reflecting the properties of the existential quantifier in free logic. This variety is generated by a certain class FMBA of algebras isomorphic to ones whose elements are propositional functions.

We show that FMBA is characterised by the disjunction of the equations $\exists E=\mathbf{1}$ and $\exists E=\mathbf{0}$. We also define a weaker notion of "relatively functional" algebra, and show that every member of MBA is isomorphic to a relatively functional one.
\end{abstract}

In [1], an equationally defined class MBA of monadic bounded algebras was introduced. Each of these algebras comprises a Boolean algebra $\mathbf{B}$ with a distinguished element $E$, thought of as an existence predicate, and an operator $\exists$ on $\mathbf{B}$ reflecting the properties of the existential quantifier in logic without existence assumptions. MBA was shown to be generated by a certain proper subclass FMBA of algebras isomorphic to algebras of Boolean-valued functions.

In this paper we characterise FMBA as consisting precisely of those monadic bounded algebras in which $\exists E=\mathbf{0}$ or $\exists E=\mathbf{1}$. So FMBA is defined by a disjunction of two equations. We also define a weaker notion of "relativised" functional algebra and show that every monadic bounded algebra is isomorphic to one of these more general functional ones. The paper builds on [1], with which the reader is assumed to be familiar.

We review the definition of FMBA. Let $\mathbf{B}$ be a Boolean algebra, $X$ a set, and $X_{E} \subseteq X$. The set $\mathbf{B}^{X}$ of all functions from $X$ to $\mathbf{B}$ is a Boolean algebra with respect to the pointwise operations. A Boolean subalgebra $\mathbf{A}$ of $\mathbf{B}^{X}$ with a distinguished member $E$ of $\mathbf{A}$ is called a functional monadic bounded algebra, with domain $\left(X, X_{E}\right)$ and distinguished function $E$, or more briefly a functional MBA, iff

(F1) $E(x)=\mathbf{1}^{\mathbf{B}}$ for every $x \in X_{E}$;

(F2) for every $p \in \mathbf{A}$, both $\bigvee\left\{p(x) \mid x \in X_{E}\right\}$ and $\bigvee\{p(x) \wedge E(x) \mid x \in X\}$ exist in $\mathbf{B}$ and are equal; and

(F3) for every $p \in \mathbf{A}, \mathbf{A}$ contains the constant function $\exists p$ on $X$, defined by 


$$
\exists p(y)=\bigvee\left\{p(x) \mid x \in X_{E}\right\}
$$

FMBA is the class of all algebras that are isomorphic to some functional algebra meeting this definition. MBA on the other hand is a class of abstract algebras $\mathbf{A}=(\mathbf{B}, E, \exists)$ satisfying the equational conditions $(\operatorname{ax} 1)-(\operatorname{ax} 6)$ stated in $[1$, $\S 3]$. In $\S 6$ of [1] there is an example of a monadic bounded algebra that is not isomorphic to any functional one.

Note that if $\mathbf{A}$ is a functional $\mathbf{M B A}$ as above, and $\mathbf{A}^{*}$ is any subalgebra of $\mathbf{A}$, hence $\mathbf{A}^{*}$ contains $E$ and is closed under $\exists$, then (F2) and (F3) remain true for all $p \in \mathbf{A}^{*}$, so $\mathbf{A}^{*}$ is also a functional MBA with the same domain and distinguished element.

To build functional algebras we need the notion of a constant from the theory of monadic algebras [2, p. 63]. This is motivated by the question of how to represent the concept of a particular individual $x_{0} \in X$ within the structure of an abstract algebra. Think of the process of applying each predicate (like "is human") to $x_{0}$ to form a proposition (" $x_{0}$ is human"). Since predicates correspond to propositional functions $p: X \rightarrow \mathbf{B}$, this suggests the definition of a function $c: \mathbf{B}^{X} \rightarrow \mathbf{B}^{X}$ assigning to each $p \in \mathbf{B}^{X}$ the function $c p$ defined by $c p(x)=p\left(x_{0}\right)$. This $c$ is an endomorphism of the Boolean algebra $\mathbf{B}^{X}$. If $\mathbf{B}^{X}$ is a functional MBA with respect to $E$ and $\exists$, then $c \circ \exists=\exists$, since $c(\exists p)(x)=\exists p\left(x_{0}\right)=\exists p(x)$ in general. Note also that if $x_{0} \in X_{E}$, then $c E=\mathbf{1}$ in $\mathbf{B}^{X}$, as $c E(x)=E\left(x_{0}\right)=\mathbf{1}$ in $\mathbf{B}$.

Now let $\mathbf{A}=(\mathbf{B}, E, \exists)$ be an abstract MBA. A constant of $\mathbf{A}$ is defined to be a Boolean homomorphism $c: \mathbf{B} \rightarrow \mathbf{B}$ such that $c \circ \exists=\exists$. Note that the identity function on $\mathbf{B}$ is a constant, a fact we make significant use of. ${ }^{1}$ Let $X^{\mathbf{A}}$ be the set of all constants on $\mathbf{A}$, and

$$
X_{E}^{\mathbf{A}}=\left\{c \in X^{\mathbf{A}} \mid c E=\mathbf{1}^{\mathbf{A}}\right\} .
$$

A will be called a rich algebra if it satisfies:

(R1) if $p \wedge E \neq \mathbf{0}$, then there is a $c \in X_{E}^{\mathbf{A}}$ with $\exists p=c p$.

(R2) if $p \neq \mathbf{0}$, then there is a $c \in X^{\mathbf{A}}$ with $c p \neq \mathbf{0}$.

If $\mathbf{A}$ is a monadic algebra, then $E=\mathbf{1}, X_{E}^{\mathbf{A}}=X^{\mathbf{A}}$ and $p \leq \exists p$, so (R2) follows from (R1). So our definition of rich is consistent with that of [2].

Theorem 1. Every rich MBA is isomorphic to a functional MBA.

Proof. Let $\mathbf{A}$ be an MBA with $X^{\mathbf{A}}$ and $X_{E}^{\mathbf{A}}$ as above. For each $p \in \mathbf{A}$, define $\widetilde{p}: X^{\mathbf{A}} \rightarrow \mathbf{B}$ by putting $\widetilde{p}(c)=c p$. Then define a function $f$ from $\mathbf{B}$ to $\mathbf{B}^{X^{\mathbf{A}}}$ by putting $f(p)=\widetilde{p}$. It is readily checked that $f$ is a Boolean homomorphism,

\footnotetext{
${ }^{1}$ Constants on a monadic algebra in [2] are also required to satisfy the condition $\exists \circ c=c$, which would exclude the identity as $c$ unless $\exists$ is the identity. We do not need this condition, and it can fail in the example of the constant defined by $x_{0}$ above, e.g. if $X_{E}=\emptyset$. For that example we have only $\exists c p \leq c p$, and $\exists c p \wedge E=c p \wedge E$.
} 
because each constant preserves the Boolean operations in $\mathbf{B}$, and these operations are defined pointwise in the functional algebra $\mathbf{B}^{X^{\mathbf{A}}}$. Also $f$ is injective by the condition (R2), which implies that if $p \neq \mathbf{0}$ in $\mathbf{B}$, then there exists $c \in X^{\mathbf{A}}$ with $\widetilde{p}(c) \neq \mathbf{0}$, hence $f(p) \neq \mathbf{0}$ in $\mathbf{B}^{X^{\mathbf{A}}}$.

Now let $\widetilde{\mathbf{A}}$ be the range of $f$, a subalgebra of $\mathbf{B}^{X^{\mathbf{A}}}$ that is isomorphic to $\mathbf{B}$, and contains $\widetilde{E}$. We will demonstrate that $\widetilde{\mathbf{A}}$ is a functional MBA with domain $\left(X^{\mathbf{A}}, X_{E}^{\mathbf{A}}\right)$ and distinguished function $\widetilde{E}$ that is isomorphic to $\mathbf{A}$.

For condition (F1) in the definition of a functional MBA, if $c \in X_{E}^{\mathbf{A}}$ then since $c E=1^{\mathrm{B}}$ it is immediate that $\widetilde{E}(c)=1^{\mathrm{B}}$ as required. For (F2) and (F3) we show that for each $p$ in $\mathbf{A}$,

$$
\exists p=\bigvee\left\{\widetilde{p}(c) \mid c \in X_{E}^{\mathbf{A}}\right\}=\bigvee\left\{\widetilde{p}(c) \wedge \widetilde{E}(c) \mid x \in X^{\mathbf{A}}\right\}
$$

in $\mathbf{B}$, or equivalently that

$$
\exists p=\bigvee_{c \in X_{E}^{\mathrm{A}}} c p=\bigvee_{c \in X^{\mathrm{A}}} c(p \wedge E)
$$

This ensures that (F2) holds for each $\widetilde{p} \in \widetilde{\mathbf{A}}$. Since $\widetilde{\exists}(c)=c(\exists p)=\exists p$ (as $c \circ \exists=\exists$ ), it also ensures that $\widetilde{\exists}$ is the function $\exists \widetilde{p}$ on $X^{\mathbf{A}}$ with constant value $\bigvee\left\{\widetilde{p}(c) \mid c \in X_{E}^{\mathbf{A}}\right\}$, and hence that this function belongs to $\widetilde{\mathbf{A}}$, giving (F3). That makes $\widetilde{\mathbf{A}}$ a functional MBA. But then $f(\exists p)=\widetilde{\exists p}=\exists \widetilde{p}=\exists f(p)$, and $f(E)=\widetilde{E}$, so $f$ is an MBA-homomorphism making $\mathbf{A}$ isomorphic to $\widetilde{\mathbf{A}}$, completing the proof.

It remains to prove (1). We note that

(i) If $c \in X^{\mathbf{A}}$, then $c(p \wedge E) \leq \exists p ; \quad$ and

(ii) If $c \in X_{E}^{\mathbf{A}}$, then $c p=c(p \wedge E)$.

(i) holds as $p \wedge E \leq \exists p$ by (ax3), and $c$ is monotonic, so $c(p \wedge E) \leq c \exists p$; but $c \exists p=\exists p$ as $c$ is a constant of $\mathbf{A}$. (ii) holds because $c E=\mathbf{1}$, so $c p=c p \wedge \mathbf{1}=$ $c p \wedge c E=c(p \wedge E)$.

There are two cases for (1). The first is that $p \wedge E=\mathbf{0}$, i.e. $p \leq E^{\prime}$. Recall from [1] that $\exists$ takes the value $\mathbf{0}$ on the ideal generated by $E^{\prime}$ in any MBA, so $\exists p=\mathbf{0}$ here. Hence for any $c \in X^{\mathbf{A}}$, we get $c(p \wedge E)=\mathbf{0}$ by (i). But then if $c \in X_{E}^{\mathbf{A}}$, we get $c p=\mathbf{0}$ by (ii). So (1) holds in this case because all elements referred to in (1) are equal to $\mathbf{0}$.

The other case is when $p \wedge E \neq \mathbf{0}$. Then by richness condition (R1) for $\mathbf{A}$, there is some $c^{*} \in X_{E}^{\mathbf{A}}$ with $\exists p=c^{*} p$. Now (ii) implies that

$$
\left\{c p \mid c \in X_{E}^{\mathbf{A}}\right\} \subseteq\left\{c(p \wedge E) \mid c \in X^{\mathbf{A}}\right\} .
$$

(i) states that $\exists p$ is an upper bound of the larger of these two sets. But $\exists p$ is $c^{*} p$, which belongs to the smaller set. Thus $\exists p$ belongs to both sets and is an upper bound of both, hence is the least upper bound of both, i.e. (1) holds. 
This proof provides the additional information that for any $q \in \widetilde{\mathbf{A}}$,

if $q \wedge \widetilde{E} \neq \mathbf{0}$, then there is some $c \in X_{E}^{\mathbf{A}}$ with $q(c)=$ the constant value of $\exists q$.

For if $q=\widetilde{p}$ and $q \wedge \widetilde{E} \neq \mathbf{0}$ in $\widetilde{\mathbf{A}}$, then $p \wedge E \neq \mathbf{0}$ in $\mathbf{A}$, so by (R1) there is some $c \in X_{E}^{\mathbf{A}}$ with $c p=\exists p$, which says that $\widetilde{p}(c)=\exists \widetilde{p}(c)$.

We turn now to results about the existence of richness. First we show that when $\exists E=\mathbf{1}$, then condition (R1) can be strengthened.

Lemma 2. Let A be a rich MBA having $\exists E=\mathbf{1}$. Then for every element $p$ of $\mathbf{A}$, there is some constant $c$ of $\mathbf{A}$ with $c E=\mathbf{1}$ and $\exists p=c p$.

Proof. If $E=\mathbf{0}$, then $\mathbf{1}=\exists E=\exists \mathbf{0}=\mathbf{0}$. Hence $\mathbf{A}$ is a one-element algebra, and the conclusion of the Lemma holds simply by taking $c$ as the identity function on $\mathbf{A}$.

So we may assume $E \neq \mathbf{0}$. Then putting $p=E$ in (R1), there is some $c \in X_{E}^{\mathbf{A}}$ with $c E=\exists E=\mathbf{1}$. Hence $c\left(E^{\prime}\right)=(c E)^{\prime}=\mathbf{0}$. Now for any $p \in \mathbf{A}$, if $p \wedge E=\mathbf{0}$, then $p \leq E^{\prime}$, so $\exists p=\mathbf{0}$ and $c p \leq c\left(E^{\prime}\right)=\mathbf{0}$, giving $\exists p=c p(=\mathbf{0})$ to fulfil the Lemma in this case.

But if $p \wedge E \neq \mathbf{0}$, the desired conclusion is directly given by (R1).

Theorem 3. If $\left\{\mathbf{A}_{i} \mid i \in I\right\}$ is a collection of rich MBA's that satisfy $\exists E=\mathbf{1}$, then the direct product $\prod_{I} \mathbf{A}_{i}$ is rich.

Proof. Let $\mathbf{A}_{i}=\left(\mathbf{B}_{i}, E_{i}, \exists_{i}\right)$ with greatest and least elements $\mathbf{1}_{i}$ and $\mathbf{0}_{i}$, so $\exists_{i} E_{i}=\mathbf{1}_{i}$. Let $\mathbf{A}$ be $\prod_{I} \mathbf{A}_{i}$. For each $i$, let $\pi_{i}: \mathbf{A} \rightarrow \mathbf{A}_{i}$ be the projection homomorphism, and write $p_{i}=\pi_{i}(p)$ for each $p \in \mathbf{A}$. Then $p$ is the tuple $\left\langle p_{i} \mid i \in I\right\rangle$. In particular, the distinguished element $E$ of $\mathbf{A}$ is $\left\langle E_{i} \mid i \in I\right\rangle$.

To prove A satisfies (R1), we prove the stronger version from Lemma 2. Let $p \in \mathbf{A}$. Then for each $i \in I$, by the Lemma applied to $\mathbf{A}_{i}$ there is some constant $c_{i}$ of $\mathbf{A}_{i}$ with $c_{i} E_{i}=\mathbf{1}_{i}$ and $\exists_{i} p_{i}=c_{i} p_{i}$. Let $c: \mathbf{A} \rightarrow \mathbf{A}$ be the product of all these $c_{i}$ 's, defined by $(c q)_{i}=c_{i}\left(q_{i}\right)$. Then $c$ is a Boolean homomorphism, as each $c_{i}$ is. Also $(c \exists q)_{i}=c_{i} \exists_{i} q_{i}=\exists_{i} q_{i}$ for all $i \in I$, so $c \exists q=\exists q$ in general. Hence $c$ is a constant of $\mathbf{A}$. Similarly $(c E)_{i}=c_{i} E_{i}=\mathbf{1}_{i}$ for all $i$, so $c E=\mathbf{1}$ in A. Finally, $(\exists p)_{i}=\exists_{i} p_{i}=c_{i} p_{i}=(c p)_{i}$ in general, so $\exists p=c p$ as required.

To prove $\mathbf{A}$ satisfies (R2), let $p \neq \mathbf{0}$. Then for some $i, p_{i} \neq \mathbf{0}_{i}$, so by (R2) in $\mathbf{A}_{i}$, there is some constant $c_{i}$ of $\mathbf{A}_{i}$ with $c_{i} p_{i} \neq \mathbf{0}_{i}$. For each $j \neq i$, let $c_{j}$ be the identity constant on $\mathbf{A}_{j}$. Then let $c$ be the product of $\left\{c_{j} \mid j \in I\right\}$. As above, $c$ is a constant on $\mathbf{A}$. But $(c p)_{i}=c_{i} p_{i} \neq \mathbf{0}_{i}$, so $c p \neq \mathbf{0}$ as required.

Theorem 4. Every basic MBA is rich.

Proof. Recall that in a basic MBA, the quantifier takes the value $\mathbf{1}$ outside the ideal $\left\{p \mid p \leq E^{\prime}\right\}$. In any MBA it takes the value $\mathbf{0}$ on this ideal, as noted earlier.

It follows that if $\mathbf{A}$ is basic, then any Boolean homomorphism $c: \mathbf{A} \rightarrow \mathbf{A}$ is a constant of $\mathbf{A}$, since $\exists p \in\{\mathbf{0}, \mathbf{1}\}$ and $c$ fixes $\mathbf{0}$ and $\mathbf{1}$, so $c \exists p=\exists p$ for all $p$. In 
particular, if $U$ is an ultrafilter of $\mathbf{A}$, then the characteristic function of $U$, of the form $\mathbf{A} \rightarrow\{\mathbf{0}, \mathbf{1}\} \subseteq \mathbf{A}$, is a constant of $\mathbf{A}$.

To prove (R1) for $\mathbf{A}$, suppose $p \wedge E \neq \mathbf{0}$. Then there is an ultrafilter $U$ of A with $p \wedge E \in U$. Let $c$ be the characteristic function of $U$. Then $E \in U$, so $c E=\mathbf{1}$ and hence $c \in X_{E}^{\mathbf{A}}$. Also $p \in U$, so $c p=\mathbf{1}$. But $\exists p=\mathbf{1}$ as $\mathbf{A}$ is basic, so $c p=\exists p$ as required.

For (R2), if $p \neq \mathbf{0}$, there is an ultrafilter $U$ with $p \in U$. Again let $c$ be the characteristic function of $U$. Then $c \in X^{\mathbf{A}}$ and $c p=\mathbf{1}$. But $p \neq \mathbf{0}$ implies $\mathbf{1} \neq \mathbf{0}$, so $c p \neq \mathbf{0}$ as required.

We are now ready to prove our main result.

Theorem 5. FMBA is precisely the class of all monadic bounded algebras in which $\exists E$ is $\mathbf{0}$ or $\mathbf{1}$.

Proof. Theorem 2.3 of [1] showed that every functional MBA has $\exists E \in\{\mathbf{0}, \mathbf{1}\}$, hence so does every algebra isomorphic to a functional MBA, i.e. every member of FMBA.

For the converse, let $\mathbf{A}=\left(\mathbf{B}, E^{\mathbf{A}}, \exists^{\mathbf{A}}\right)$ be any MBA having $\exists E^{\mathbf{A}} \in\left\{\mathbf{0}^{\mathbf{A}}, \mathbf{1}^{\mathbf{A}}\right\}$. If in fact $\exists E^{\mathbf{A}}=\mathbf{0}^{\mathbf{A}}$, then $E^{\mathbf{A}}=\mathbf{0}^{\mathbf{A}}$, and $\exists^{\mathbf{A}} p=\mathbf{0}^{\mathbf{A}}$ for all $p$. By the Stone representation of $\mathbf{B}$ there is a set $X$ and a Boolean monomorphism $f: \mathbf{B} \rightarrow \mathbf{2}^{X}$ making $\mathbf{B}$ isomorphic to a subalgebra $\widetilde{\mathbf{A}}$ of the functional Boolean algebra $\mathbf{2}^{X}$. Let $E=f E^{\mathbf{A}}=f \mathbf{0}^{\mathbf{A}}=\mathbf{0}$ in $\mathbf{A}$. Put $X_{E}=\emptyset$. Then it is readily checked that $\widetilde{\mathbf{A}}$ is a functional MBA with domain $\left(X, X_{E}\right)$ and distinguished function $E$. The condition (F1) holds vacuously as $X_{E}=\emptyset$. For each $p$ in $\mathbf{A}$, the sets $\bigvee\left\{f p(x) \mid x \in X_{E}\right\}$ and $\bigvee\{f p(x) \wedge E(x) \mid x \in X\}$ both have join $\mathbf{0}^{\mathbf{B}}$, and the function $\exists f p$ on $X$ defined by $\exists f p(y)=\bigvee\left\{f p(x) \mid x \in X_{E}\right\}$ has constant value $\mathbf{0}^{\mathbf{B}}$, so is equal to $f \mathbf{0}^{\mathbf{A}} \in \widetilde{\mathbf{A}}$. This proves (F2) and (F3) for $\widetilde{\mathbf{A}}$. But also $f \exists^{\mathbf{A}} p=f \mathbf{0}^{\mathbf{A}}=\exists f p$, so $f$ is an $\mathbf{M B A}$-homomorphism making $\mathbf{A}$ isomorphic to the functional MBA $\widetilde{\mathbf{A}}$.

Alternatively, $\exists E^{\mathbf{A}}=\mathbf{1}^{\mathbf{A}}$. Now by Theorem 5.1 of [1], every MBA is isomorphic to a subdirect product of basic MBA's. Hence there is a collection $\left\{\mathbf{A}_{i} \mid i \in I\right\}$ of basic MBA's and an injective homomorphism $f: \mathbf{A} \rightarrow \prod_{i \in I} \mathbf{A}_{i}$. For each $i \in I$, composing $f$ with the projection from $\prod_{i \in I} \mathbf{A}_{i}$ shows there is a homomorphism $\mathbf{A} \rightarrow \mathbf{A}_{i}$, implying $\exists_{i} E_{i}=\mathbf{1}_{i}$. Also $\mathbf{A}_{i}$ is rich by Theorem 4. Hence by Theorem $3, \prod_{i \in I} \mathbf{A}_{i}$ is rich, and so is isomorphic to some functional MBA $\widetilde{\mathbf{A}}$ by Theorem 1 . Let $\widetilde{f}$ be the composition of $f$ with the isomorphism from $\prod_{i \in I} \mathbf{A}_{i}$ to $\widetilde{\mathbf{A}}$. The range of $\widetilde{f}$ is then a subalgebra of $\widetilde{\mathbf{A}}$, hence a functional MBA with the same domain and distinguished element as $\widetilde{\mathbf{A}}$, to which A is isomorphic under $\widetilde{f}$.

So in both cases we get that $\mathbf{A}$ is isomorphic to a functional MBA and hence belongs to FMBA.

The other topic of this paper is the development of a weaker notion of functional algebra, in terms of which every MBA can be represented. Given a set $X$ and a Boolean algebra $\mathbf{B}$, then a Boolean subalgebra $\mathbf{A}$ of $\mathbf{B}^{X}$ with a distinguished element $E$ is called a relatively functional MBA if for each $p \in \mathbf{A}$, 
the join $\bigvee\{p(x) \wedge E(x) \mid x \in X\}$ exists in $\mathbf{B}$, and $\mathbf{A}$ contains the constant function $\exists p$ on $X$ with this join as value. In this definition, we have abandoned the notion of the set $X_{E}$, but have retained enough structure to ensure that $\mathbf{A}$ is an MBA.

Note that any subalgebra of a relatively functional MBA is a relatively functional MBA with the same distinguished element.

One way to obtain algebras of this kind is to apply the notion of relativised monadic algebra from Example 3.1 of [1]. A functional monadic algebra based on $X$ and $\mathbf{B}$ is a Boolean subalgebra $\mathbf{A}$ of $\mathbf{B}^{X}$ such that for every $p \in \mathbf{A}$, the join $\bigvee\{p(x) \mid x \in X\}$ exists in $\mathbf{B}$, and $\mathbf{A}$ contains the constant function $\exists p$ on $X$ with this join as value. Here we have no $E$ as well as no $X_{E}$. Any monadic algebra (i.e. any MBA with $E=\mathbf{1}$ ) is isomorphic to such a functional monadic algebra [2, p. 70]. But if $\mathbf{A}$ is a functional monadic algebra as described, and $E$ is an arbitrary element of $\mathbf{A}$, we can define an operation $\exists^{E}$ on $\mathbf{A}$ by putting $\exists^{E} p=\exists(p \wedge E) \in \mathbf{A}$ for all $\in \mathbf{A}$. Then for any $y \in X$ we have

$$
\exists^{E} p(y)=\bigvee\{p(x) \wedge E(x) \mid x \in X\} \text { in } \mathbf{B} .
$$

So this creates from $\mathbf{A}$ a relatively functional $\mathbf{M B A} \mathbf{A}^{E}$ with distinguished element $E$ and quantifier $\exists^{E}$. The notion of relatively functional MBA is itself more general than this, as it does not assume the existence of any background functional monadic algebra.

Next we define an abstract MBA A to be relatively rich if it satisfies richness condition (R2), and in place of (R1) it has

(R1') for any $p$ there is a $c \in X^{\mathbf{A}}$ with $\exists p=c(p \wedge E)$.

Lemma 6. Every rich MBA is relatively rich.

Proof. Let A satisfy (R1) and (R2). To prove (R1'), suppose first that $p \wedge E \neq \mathbf{0}$. Then by (R1) there is a $c \in X_{E}^{\mathbf{A}}$ with $\exists p=c p$. But $c E=\mathbf{1}$, so $c(p \wedge E)=$ $c p \wedge c E=c p=\exists p$.

But if $p \wedge E=\mathbf{0}$, i.e. $p \leq E^{\prime}$, then $\exists p=\mathbf{0}$. Let $c \in X^{\mathbf{A}}$ be the identity constant on $\mathbf{A}$. Then $c(p \wedge E)=c \mathbf{0}=\mathbf{0}=\exists p$.

Theorem 7. Any direct product of relatively rich MBA's is relatively rich.

Proof. Let $\mathbf{A}=\prod_{i \in I} \mathbf{A}_{i}$ with each $\mathbf{A}_{i}$ relatively rich. We use the notation of the proof of Theorem 3. For each $p \in \mathbf{A}$ and each $i \in I$, by (R1') in $\mathbf{A}_{i}$ there is a constant $c_{i}$ on $\mathbf{A}_{i}$ with $\exists p_{i}=c_{i}\left(p_{i} \wedge E_{i}\right)$. Let $c$ be the product of these $c_{i}$ 's. Then $c$ is a constant on $\mathbf{A}$, as in Theorem 3, with $\exists p=c(p \wedge E)$.

This shows that $\mathbf{A}$ satisfies $\left(\mathrm{R} 1^{\prime}\right)$. The proof that it satisfies (R2) is unchanged from Theorem 3 .

Theorem 8. Every relatively rich MBA is isomorphic to a relatively functional MBA. 
Proof. Let $\mathbf{A}=(\mathbf{B}, E, \exists)$ be relatively rich. We repeat the construction used in Theorem 1. For each $p \in \mathbf{A}$, define $\widetilde{p}: X^{\mathbf{A}} \rightarrow \mathbf{B}$ by putting $\widetilde{p}(c)=c p$; and then $f: \mathbf{B} \rightarrow \mathbf{B}^{X^{\mathbf{A}}}$ by $f(p)=\widetilde{p}$. Let $\widetilde{\mathbf{A}}$ be the range of $f . f$ is a Boolean homomorphism, and is injective because $\mathbf{A}$ satisfies (R2).

To show that $\widetilde{\mathbf{A}}$ is a relatively functional MBA with distinguished function $\widetilde{E}$, it suffices to show that for each $p$ in $\mathbf{A}$,

$$
\exists p=\bigvee\left\{\widetilde{p}(c) \wedge \widetilde{E}(c) \mid x \in X^{\mathbf{A}}\right\}
$$

in $\mathbf{B}$, i.e. that

$$
\exists p=\bigvee_{c \in X^{\mathbf{A}}} c(p \wedge E) .
$$

This ensures that $\widetilde{\exists p}$ is the function $\exists \widetilde{p}$ on $X^{\mathbf{A}}$ with constant value $\bigvee\{\widetilde{p}(c) \wedge \widetilde{E}(c) \mid$ $\left.x \in X^{\mathbf{A}}\right\}$, and hence that this function belongs to $\widetilde{\mathbf{A}}$. Then $f(\exists p)=\exists f(p)$, and $f$ is an MBA-homomorphism making $\mathbf{A}$ isomorphic to the relatively functional algebra $\widetilde{\mathbf{A}}$, completing the proof.

To prove (3), note that for a given $p$, by $\left(\mathrm{R} 1^{\prime}\right)$ there is some $c^{*} \in X^{\mathbf{A}}$ with $\exists p=c^{*}(p \wedge E)$. But for all $c \in X^{\mathbf{A}}$, we have $c(p \wedge E) \leq c \exists p=\exists p$. Thus $\exists p$ is an upper bound of $\left\{c(p \wedge E) \mid c \in X^{\mathbf{A}}\right\}$ and also belongs to this set, which implies (3).

We can now show that these functional algebras encompass all monadic bounded algebras.

Theorem 9. Every MBA is isomorphic to a relatively functional MBA.

Proof. If $\mathbf{A}$ is any MBA, by [1, Theorem 5.1] there is an injective homomorphism $f: \mathbf{A} \rightarrow \prod_{i \in I} \mathbf{A}_{i}$ into a direct product for which every $\mathbf{A}_{i}$ is basic, hence rich, hence relatively rich (Lemma 6). Then $\prod_{i \in I} \mathbf{A}_{i}$ is relatively rich (Theorem 7 ), so is isomorphic to a relatively functional MBA $\widetilde{\mathbf{A}}$ (Theorem 8). Let $\widetilde{f}$ be the composition of $f$ with this isomorphism from $\prod_{i \in I} \mathbf{A}_{i}$ to $\widetilde{\mathbf{A}}$. The range of $\widetilde{f}$ is then a subalgebra of $\widetilde{\mathbf{A}}$, hence a relatively functional MBA with the same distinguished element as $\widetilde{\mathbf{A}}$, to which $\mathbf{A}$ is isomorphic under $\widetilde{f}$.

\section{References}

[1] Galym Akishev and Robert Goldblatt. Monadic bounded algebras.

[2] Paul R. Halmos. Algebraic Logic. Chelsea, New York, 1962. 